\title{
Particle rearrangements during transitions between local minima of the potential energy landscape of a binary Lennard-Jones liquid
}

\author{
Michael Vogel $\left.\right|^{\mathrm{a})}$ \\ Department of Chemical Engineering, University of Michigan, Ann Arbor, Michigan 48109 \\ Burkhard Doliwa \\ Max Planck Institute for Polymer Research, Postfach 3148, 55021 Mainz, Germany \\ Andreas Heuer \\ Institute of Physical Chemistry, University of Münster, Schlossplatz 4/7, 48149 Münster, Germany \\ Sharon C. Glotzer \\ Departments of Chemical Engineering and Materials Science and Engineering, University of Michigan, \\ Ann Arbor, Michigan 48109
}

(Received 15 May 2003; accepted 4 December 2003)

\begin{abstract}
The potential energy landscape (PEL) of binary Lennard-Jones (BLJ) mixtures exhibits local minima, or inherent structures (IS), which are organized into metabasins (MBs). We study the particle rearrangements related to transitions between both successive IS and successive MB for a small 80:20 BLJ system near the mode-coupling temperature $T_{\mathrm{MCT}}$. The analysis includes the displacements of individual particles, the localization of the rearrangements, and the relevance of string-like motion. We find that the particle rearrangements during IS and MB transitions do not change significantly at $T_{\mathrm{MCT}}$. In particular, an onset of single particle hopping on the length scale of the interparticle distance is not observed. Further, it is demonstrated that IS and MB dynamics are spatially heterogeneous and facilitated by string-like motion. To investigate the mechanism of string-like motion, we follow the particle rearrangements during suitable sequences of IS transitions. We find that most strings observed after a series of transitions do not move coherently during a single transition, but subunits of different sizes are active at different times. Several findings suggest that, though string-like motion is of comparable relevance when the system explores a MB and when it moves from one MB to another, the occurrence of a successful string enables the system to exit a MB. Moreover, we show that the particle rearrangements during two consecutive MB transitions are basically uncorrelated. In particular, different groups of particles are highly mobile. We further find the positions of strings during successive MB transitions weakly but positively correlated, supporting the idea of dynamic facilitation. Finally, the relation between the features of the potential energy landscape and the relaxation processes in supercooled liquids is discussed.

(c) 2004 American Institute of Physics. [DOI: 10.1063/1.1644538]
\end{abstract}

\section{INTRODUCTION}

Potential energy landscape (PEL) approaches, which are based on the pioneering, work of Goldstein, ${ }^{1}$ have proven useful in the field of the glass transition phenomenon. ${ }^{2-4}$ In these approaches, the high-dimensional vector of all particle coordinates is considered as a point moving on the potential energy surface. A PEL description is particularly useful at sufficiently low temperatures where the evolution of the system can be decomposed into vibrations about local minima of the PEL on short time scales and transitions among distinct minima on long time scales. ${ }^{1}$ In computational work on supercooled liquids, a detailed picture of the PEL is accessible. Following Goldstein's idea, Stillinger and Weber ${ }^{5}$ introduced the concept of basins where a basin in configuration space is defined as the set of points that via steepest descent path along the PEL maps onto the same local minimum, or

\footnotetext{
a) Author to whom correspondence should be addressed. Electronic mail: mivogel@umich.edu
}

inherent structure (IS). The resulting tiling of the configuration space into nonoverlapping basins of attraction simplifies the thermodynamic description of the system. ${ }^{6,7} \mathrm{~A}$ link between thermodynamics and kinetics is suggested by the Adam-Gibbs relation which connects the relaxation times with the configurational entropy. ${ }^{8}$ Such a link was qualitatively established by Sastry et al. ${ }^{9}$ who observed that the onset of nonexponential relaxation is accompanied by the sampling of IS with progressively lower potential energy upon cooling. Later, the Adam-Gibbs equation was shown to hold quantitatively in simulations of water, ${ }^{10}$ a binary Lennard-Jones (BLJ) liquid, ${ }^{11}$ and silica. ${ }^{12}$ Further, dependence of the fragility on the shape of the PEL was reported. ${ }^{11,12}$ Such a relation was long proposed by Stillinger who suggested that strong liquids have a uniformly rough PEL, while the IS of fragile liquids are organized into metabasins (MBs) ${ }^{3,4}$ or, equivalently, funnels. ${ }^{13,14}$

Several workers studied the relation between the properties of the PEL and the trajectories of the individual particles in more detail. Schrøder et al. ${ }^{15}$ confirmed Goldstein's 
picture $^{1}$ showing that the dynamics of a BLJ liquid below the critical temperature of the mode coupling theory (MCT), ${ }^{16}$ $T_{\mathrm{MCT}}$, can be separated into local vibrations and transitions between IS. Further, string-like displacements of groups of particles were found during IS transitions similar to the motion observed in the equilibrium liquid. ${ }^{17}$ Instantaneous normal mode analysis suggests that a transition from nonactivated to activated dynamics takes place at $T_{\mathrm{MCT}} \cdot{ }^{18-22}$ However, the method to determine a correct distribution of saddle points is still a matter of debate. ${ }^{23,24}$ In different approaches, no change of the dynamical behavior at $T_{\mathrm{MCT}}$ was observed, but activated barrier crossing was found to be relevant already above $T_{\mathrm{MCT}} \cdot{ }^{24-26}$ Doliwa and Heuer ${ }^{24,25}$ calculated the diffusion constant $D(T)$ from the static properties of the PEL by taking into account the organization of the IS into MBs. In this way, the effect of back-and-forth jumps, which frequently occur between the IS within the same MB but do not contribute to long-range diffusion, can be eliminated, and only hopping between MB is considered, which may resemble a random walk on the PEL. According to Denny et al. $^{26}$ " transitions between IS within a MB involve very small flexing of a cage, while transitions between MB involve collective rearrangements." Hernandez-Rojas and Wales observed that upon cooling the distance atoms move during an IS transition decreases significantly at $T \approx T_{\mathrm{MCT}} \cdot{ }^{27}$

Despite this progress, the relation between the dynamics of single particles in real space and the motion of the system in configuration space is still elusive. In this paper, we present a detailed study of the particle rearrangements resulting from transitions between local minima of the PEL of a BLJ liquid at $T \approx T_{\mathrm{MCT}}$. Due to the organization of IS into $\mathrm{MB}$, we consider transitions between consecutive IS and consecutive MB where the latter result from a series of the former. For simplicity, we refer to the corresponding displacements as IS dynamics and MB dynamics, respectively. Stillinger ${ }^{3}$ related MB dynamics to the $\alpha$ process and IS dynamics to the (Johari-Goldstein) $\beta$ process $^{28}$ and, hence, our approach may yield valuable insights into the nature of these relaxation processes in supercooled liquids. The present analysis of IS/MB dynamics includes measures for the displacements of the individual particles, the localization of the rearrangements and the relevance of string-like motion, which is believed to facilitate the structural relaxation in supercooled liquids. ${ }^{17,29,30} \mathrm{We}$ focus on two temperatures $T_{h}>T_{\mathrm{MCT}}$ and $T_{l} \leqslant T_{\mathrm{MCT}}$. In this way, we can investigate whether a change of the transport mechanism observed for the equilibrium liquid at $T \approx T_{\mathrm{MCT}},, 15,31,32$ manifests itself in distinct IS/MB dynamics. To analyze IS/MB dynamics beyond the concept of single transitions, we study the correlation of the particle displacements during consecutive IS/MB transitions. In view of our results and recent experimental findings, we suggest a modification of Stillinger's picture of the relation between the features of the PEL and the relaxation processes in supercooled liquids.

\section{MODEL AND SIMULATION}

The simulations used to generate the data analyzed here are described in Ref. 24. In summary, we investigate a BLJ liquid characterized by the interaction potential

$$
V_{\alpha \beta}(r)=4 \epsilon_{\alpha \beta}\left[\left(\sigma_{\alpha \beta} / r\right)^{12}-\left(\sigma_{\alpha \beta} / r\right)^{6}\right]
$$

with the parameters $N=N_{A}+N_{B}=65, \quad N_{A}=52, \quad \sigma_{A B}$ $=0.8 \sigma_{A A}, \sigma_{B B}=0.88 \sigma_{A A}, \epsilon_{A B}=1.5 \epsilon_{A A}, \epsilon_{B B}=0.5 \epsilon_{A A}$, and $r_{c}=1.8$. Linear functions are added to the potentials to ensure continuous forces and energies at the cutoff $r_{c}$. These modifications of the original potential of Kob and Andersen $^{33,34}$ are necessary for the simulation of small systems. ${ }^{19,27}$ The data were generated using Langevin molecular dynamics (MD) simulations with fixed step size $\lambda$ $=0.015=\left(2 k_{B} T \Delta t / m \zeta\right)^{1 / 2}$, equal particle masses $m$, friction constant $\zeta=1$, and periodic boundary conditions. All results are reported in units of $\sigma_{A A}, m, \epsilon_{A A}$, and $m \zeta \lambda^{2} / 2 \epsilon_{A A}$ for length, mass, energy, and time, respectively.

At regularly spaced times $t_{i}$, we quench the MD trajectory $x(t)$ to the bottom of the visited basins ${ }^{24}$ yielding the discontinuous trajectory of IS, $\xi\left(t_{i}\right) .{ }^{5}$ In the IS trajectory, the entries at subsequent $t_{i}$ are identical until a transition to a new basin of attraction takes place. These multiple entries are eliminated so that the resulting trajectory only contains the IS separated by transitions. Further, we apply an interval bisection method described in Ref. 24 to ensure that all relevant transitions between the regular quenches are resolved. The final set of trajectories $\xi\left(t_{j}\right)$ contains all successively visited IS and consists of more than $10^{4}$ IS for the studied temperatures $T_{l}=0.435$ and $T_{h}=0.500$. From $\xi\left(t_{j}\right)$, we extract the $N$ trajectories of the individual particles, $\mathbf{r}^{i}\left(t_{j}\right)$, which form the basis of the present analysis. A straightforward algorithm is applied to construct the MB. ${ }^{24,35}$ First, all time intervals $\left[t_{j}^{f}, t_{j}^{l}\right]$ are searched where $t_{j}^{f}$ is the time of the first and $t_{j}^{l} \neq t_{j}^{f}$ the time of the last occurrence of the IS $\xi\left(t_{j}\right)$. Next, any two overlapping time intervals $\left[t_{j}^{f}, t_{j}^{l}\right] \cup\left[t_{k}^{f}, t_{k}^{l}\right]$ are combined. Strictly speaking, IS that occur exactly once in the trajectory $\xi\left(t_{j}\right)\left(t_{j}^{f}=t_{j}^{l}\right)$ form trivial MB. Here, we exclusively use the term MB for nontrivial MB. Thus, the MB transitions studied consist of several IS transitions.

For PEL approaches, it is essential to use small systems, because otherwise interesting effects are averaged out. ${ }^{35-37}$ Since large systems consist of many independent subsystems, which evolve in parallel, the relaxation steps of each subsystem are masked by the time evolution of the rest of the system. On the other hand, the system should not be too small so as to avoid significant finite size effects. As was discussed previously, ${ }^{24,38} N=65$ turns out to be a good compromise. Specifically, comparing the dynamics of systems with $N=65, N=135$, and $N=1000$, it was observed that the respective diffusion constants $D(T)$ are identical within $20 \%$ for all temperatures $T \geqslant T_{\mathrm{MCT}} \cdot{ }^{38}$ In addition, in our case, a critical temperature $T_{\mathrm{MCT}}=0.45 \pm 0.01$ was obtained from a power-law fit to $D(T)^{39}$ as compared to a value $T_{\mathrm{MCT}}$ $=0.435$ established for larger BLJ systems. ${ }^{33,34}$ Therefore, significant finite size effects are absent for $T \approx T_{\mathrm{MCT}}$, andsimilar to what is known for larger BLJ systems ${ }^{33,34}$ - the $N=65$ BLJ system can be regarded as a model of a typical supercooled liquid.

The mean waiting time in the $\mathrm{MB},\left\langle\tau_{\mathrm{MB}}(T)\right\rangle$, was calculated in previous work on the system studied. ${ }^{24,25}$ Its relation to the time constant of the $\alpha$ process, $\tau_{\alpha}$, depends on the system size. For $N=65, \tau_{\alpha} /\left\langle\tau_{\mathrm{MB}}\right\rangle \approx 30$ is found at $T_{l}$ and 


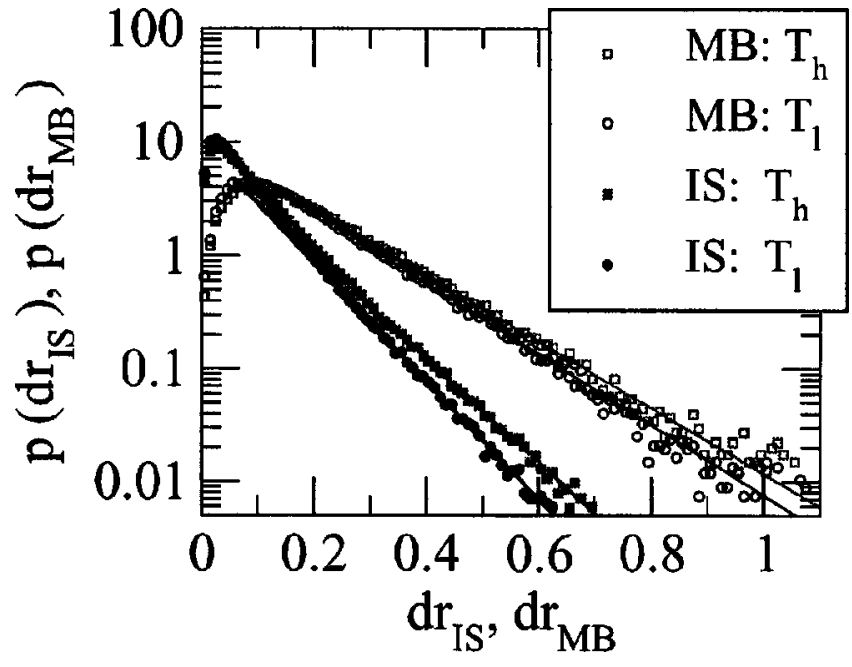

FIG. 1. Probability distributions $p\left(d r_{\mathrm{IS}}\right)$ and $p\left(d r_{\mathrm{MB}}\right)$ characterizing the displacements of the A particles during transitions between consecutive inherent structures and consecutive metabasins, respectively. Data for $T_{l}$ $=0.435$ and $T_{h}=0.500$ are compared. (Lines) Exponential decays obtained by interpolating the data at $d r_{\mathrm{IS} / \mathrm{MB}} \geqslant 0.3$.

$T_{h}$. The mean waiting times in the IS, $\left\langle\tau_{\mathrm{IS}}(T)\right\rangle$, are approximately a factor of 6 shorter than the corresponding $\left\langle\tau_{\mathrm{MB}}(T)\right\rangle$. For the diffusion constants, a ratio $D\left(T_{h}\right) / D\left(T_{l}\right)$ $\approx 10$ was reported. ${ }^{24,25}$ Despite such a moderate variation of the diffusion constant, a significant change of the transport mechanism was observed for various models of supercooled liquids in a comparable temperature range. Specifically, a secondary peak develops in the van Hove correlation function upon cooling $T \rightarrow T_{\mathrm{MCT}}$, which is usually interpreted as an onset of single-particle hopping. ${ }^{9,15,31,32}$

\section{RESULTS}

\section{A. Transitions between inherent structures}

First, we study the particle displacements $d r_{\text {IS }}$ resulting from IS transitions, i.e., the displacement of particle $i$ during the transition $\xi\left(t_{j}\right) \rightarrow \xi\left(t_{j+1}\right)$ is given by $d r_{\mathrm{IS}}=\mid \mathbf{r}^{i}\left(t_{j+1}\right)$ $-\mathbf{r}^{i}\left(t_{j}\right) \mid$. In Fig. 1, the probability distribution $p\left(d r_{\mathrm{IS}}\right)$ for the A particles of the BLJ liquid is displayed. For both temperatures, a rapid decay dominates the distributions. Specifically, for sufficiently large displacements, $p\left(d r_{\mathrm{IS}}\right)$ decays exponentially. Thus, the distributions for $T>T_{\mathrm{MCT}}$ and $T$ $\leqslant T_{\text {MCT }}$ do not exhibit a striking difference, but the temperature dependence manifests itself in variations of the mean particle displacement $\quad\left(\left\langle d r_{\text {IS }}\left(T_{l}\right)\right\rangle=0.081, \quad\left\langle d r_{\text {IS }}\left(T_{h}\right)\right\rangle\right.$ $=0.093)$. These findings are consistent with those of Schrøder et $_{\text {al. }}{ }^{15}$ on a 50:50 BLJ mixture $(N=800)$ where $p\left(d r_{\mathrm{IS}}\right)$ for $T \approx T_{\mathrm{MCT}}$ was described by a power-law $d r_{\mathrm{IS}}^{-5 / 2}$ at small $d r_{\mathrm{IS}}$ and by an exponential decay at large $d r_{\mathrm{IS}}$. There, it was concluded that the latter functional form results from particles taking part in a local event, whereas the former is caused by particles adjusting to this event. Consistent with this interpretation, the deviations from an exponential behavior at small $d r_{\text {IS }}$ are less pronounced in our case of a smaller system, supporting the idea that our $N=65$ system resembles an indepent subsystem of a larger BLJ liquid. In contrast to Schrøder et al. ${ }^{15}$ we-except for an analysis of

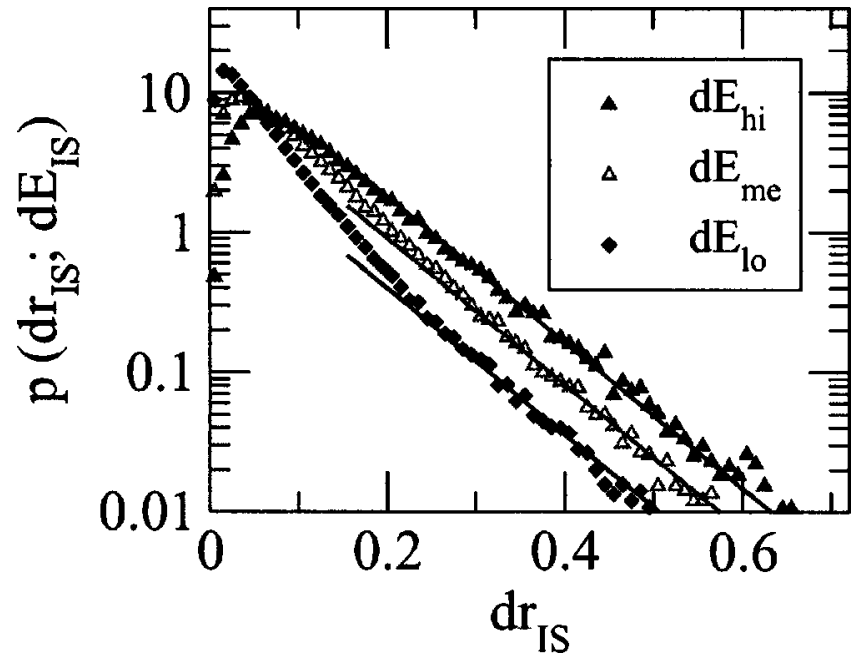

FIG. 2. Probability distributions $p\left(d r_{\mathrm{IS}} ; d E_{\mathrm{IS}}\right)$ describing the displacements of the A particles during transitions between consecutive inherent structures with energy difference $d E_{\mathrm{IS}}(T=0.435)$. We distinguish between energy differences $\quad d E_{\mathrm{IS}}<0.5\left(d E_{\mathrm{lo}}\right), \quad 0.5 \leqslant d E_{\mathrm{IS}}<1.5\left(d E_{\mathrm{me}}\right) \quad$ and $\quad 1.5$ $\leqslant d E_{\mathrm{IS}}\left(d E_{\mathrm{hi}}\right)$. (Lines) Exponential decays $A_{i} \exp \left(-d r_{\mathrm{IS}} / d r_{0}\right)$ where the factors $A_{i}$ were chosen to match the respective curves $p\left(d r_{\mathrm{IS}} ; d E_{\mathrm{IS}}\right)$ and $d r_{0}$ was obtained from an interpolation of $p\left(d r_{\mathrm{IS}}\right)$ (cf. Fig. 1).

string-like motion-do not include the B particles, because the dynamics of the minority component of the 80:20 mixture, though qualitatively similar, is somewhat faster ${ }^{33,34}$ so that a superposition of the respective results may disturb the underlying functional forms of the distribution functions.

The IS transitions are further characterized by the displacement of the whole configuration, $d r_{\mathrm{IS}}^{C}=\sum_{i=1}^{65} d r_{\mathrm{IS}}^{i}$, and by the absolute value of the energy difference of the involved IS, $d E_{\mathrm{IS}}$. For $T_{l}$ and $T_{h}$, we find that the probability distribution $p\left(d E_{\mathrm{IS}}\right)$ is well described by an exponential decay at sufficiently large $d E_{\mathrm{IS}}$. Moreover, the mean energy difference decreases upon cooling $\left(\left\langle d E_{\mathrm{IS}}\left(T_{l}\right)\right\rangle=0.78\right.$, $\left.\left\langle d E_{\mathrm{IS}}\left(T_{h}\right)\right\rangle=0.98\right)$. The probability distributions $p\left(d r_{\mathrm{IS}}^{C}\right)$ for the studied temperatures are broad and asymmetric. They exhibit a peak at $d r_{\mathrm{IS}}^{C} \approx 4$ and extend to $d r_{\mathrm{IS}}^{C} \approx 17$, indicating that the IS dynamics shows a large diversity. Thus, an analysis in terms of statistical quantities is necessary.

Next, the relation between the IS dynamics and the energy difference $d E_{\mathrm{IS}}$ is analyzed by means of the probability distributions $p\left(d r_{\mathrm{IS}} ; d E_{\mathrm{IS}}\right)$. In Fig. 2, we see that the mean displacement of the A particles increases with $d E_{\mathrm{IS}}$. For $T_{l}$, $\left\langle d r_{\text {IS }}\right\rangle=0.06$ and $\left\langle d r_{\text {IS }}\right\rangle=0.13$ are obtained for energy differences $d E_{\mathrm{IS}}<0.5\left(d E_{\mathrm{lo}}\right)$ and $d E \geqslant 1.5\left(d E_{\mathrm{hi}}\right)$, respectively. Thus, a large variation of the potential energy is accompanied by large particle rearrangements. To study IS dynamics in different regions of the PEL separately, we consider the probability distributions $p\left(d r_{\text {IS }} ; E_{\text {IS }}\right)$, where $E_{\text {IS }}$ is the energy of the initial IS of the transition. The results for $T_{l}$ in Fig. 3 show that the energy $E_{\text {IS }}$ weakly affects the particle displacements. Hence, IS dynamics in different regions of the PEL are comparable. We note that the results for $T_{h}$ are qualitatively similar to those shown in Figs. 2 and 3. Further, it was determined that the basic features of our findings do not depend on the specific choice of the energy limits.

It is known from both experiments ${ }^{40-42}$ and 


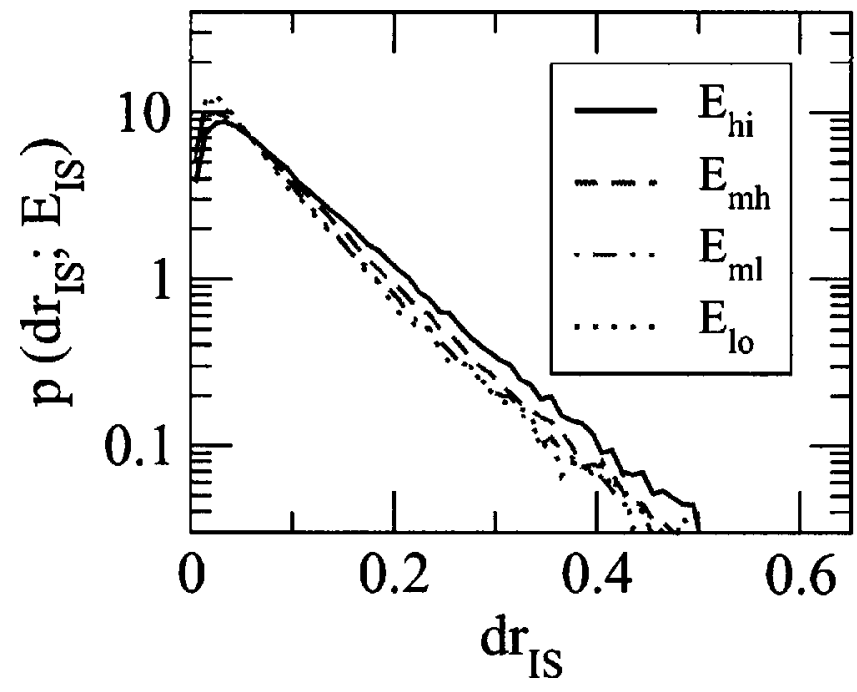

FIG. 3. Probability distributions $p\left(d r_{\mathrm{IS}} ; E_{\mathrm{IS}}\right)$ characterizing the displacement of the A particles during transitions starting from an inherent structure of energy $E_{\mathrm{IS}}$ at $T=0.435$. We differentiate between energies $E_{\mathrm{IS}}$ $<-297\left(E_{\mathrm{lo}}\right), \quad-297 \leqslant E_{\mathrm{IS}}<-295\left(E_{\mathrm{ml}}\right), \quad-295 \leqslant E_{\mathrm{IS}}<-293\left(E_{\mathrm{mh}}\right)$ and $-293 \leqslant E_{\mathrm{IS}}\left(E_{\mathrm{hi}}\right)$.

simulations ${ }^{29,30,43,44}$ that molecular dynamics in supercooled liquids are spatially heterogeneous. We analyze whether particles experiencing similar displacements due to IS transitions are spatially correlated, too, as suggested by Schrøder et al. ${ }^{15}$ Since a cluster analysis is limited by the system size $N=65$, we focus on the A particles with the largest and the smallest $d r_{\text {IS }}$ during the respective transition and analyze the mobility of their neighbors. Specifically, we first rank all particles according to their $d r_{\mathrm{IS}}$ and assign mobilities $m_{\mathrm{IS}}$ so that $m_{\mathrm{IS}}=65$ is attributed to the most mobile and $m_{\mathrm{IS}}=1$ to the most immobile particle. Then, we pick the most mobile A particle, rearrange where required the mobilities of the remaining particles $\left(m_{\mathrm{IS}}=1, \ldots, 64\right)$ and calculate the probability $p\left(m_{\mathrm{IS}}\right)$ that a particle in the first neighbor shell of the selected particle has the mobility $m_{\mathrm{IS}}$. Finally, this procedure is repeated for the most immobile A particle. Hence, if mo-

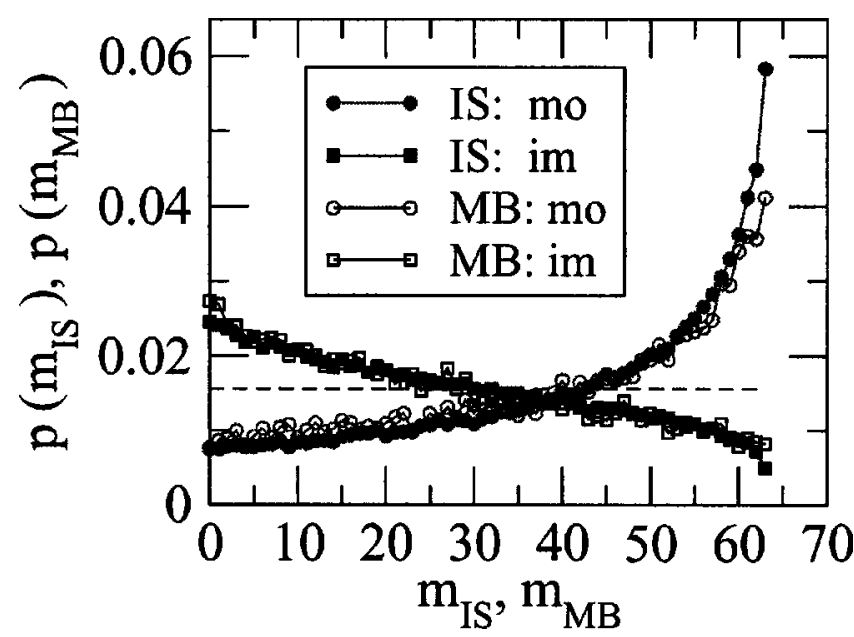

FIG. 4. Probability distributions $p\left(m_{\mathrm{IS}}\right)$ and $p\left(m_{\mathrm{MB}}\right)$ for the mobilities of the particles in the next neighbor shells of the A particles with the largest (mo) and the smallest (im) displacement during the respective inherent structure and metabasin transition. Dashed line: $p\left(m_{\mathrm{IS} / \mathrm{MB}}\right)=1 / 64$.

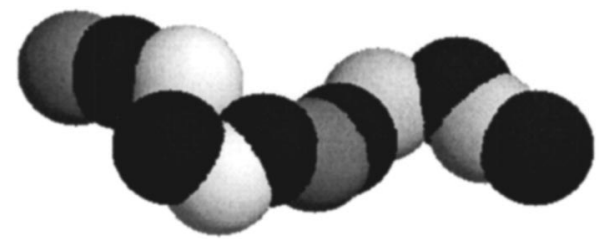

FIG. 5. Typical example of string-like motion during a sequence of transitions between inherent structures. The gray and the black spheres visualize the particles before and after the sequence, respectively. The different shades of gray denote particles replacing their neighbors at different times. It is seen that the "macrostring" after the series contains two "microstrings" due to individual transitions. The microstring on the left hand side is active first, the one on the right hand side five inherent structure transitions later.

bile and immobile particles were randomly distributed throughout the system a probability distribution $p\left(m_{\mathrm{IS}}\right)$ $=1 / 64$ would result for both the most mobile and the most immobile A particle. Figure 4 shows that a random distribution does not apply. Instead, the particles with the largest displacements are mostly surrounded by other highly mobile particles. Thus, spatially heterogeneous dynamics is also observed when analyzing IS dynamics of a 65-particle BLJ liquid close to $T_{\mathrm{MCT}}$.

For supercooled liquids close to $T_{\mathrm{MCT}}$, it has been demonstrated that the relaxation of highly mobile particles is facilitated by "string-like motion" which means that groups of particles follow each other in quasi one-dimensional paths, ${ }^{17,29,30,45,46}$ see Fig. 5. In addition, such strings were reported for the IS dynamics of a 50:50 BLJ liquid. ${ }^{15}$ Here, we quantify the contribution of this dynamical pattern to the IS dynamics in more detail. Similar to Donati et al. ${ }^{17}$ we construct strings by connecting any two particles $i$ and $k$ if $\min \left[\left|\mathbf{r}^{i}\left(t_{j}\right)-\mathbf{r}^{k}\left(t_{j+1}\right)\right|,\left|\mathbf{r}^{i}\left(t_{j+1}\right)-\mathbf{r}^{k}\left(t_{j}\right)\right|\right]<\delta=0.6, \quad$ where we now consider both the A and the B particles of the mixture. Since $\delta$ is smaller than the hard-core radii of the A and the $\mathrm{B}$ particles, this condition implies that one particle has moved and another particle has occupied its position. With this definition, string-like motion is observed during $29 \%$ of the transitions at $T_{l}$. On the other hand, for $86 \%$ of those transitions where string-like motion occurs, the most mobile particle is involved in a string. This is consistent with a spatial correlation of highly mobile particles, cf. Fig. 4.

The strings can be further characterized by their length $l$, i.e., the number of participating particles. In Fig. 6, we display the mean number of strings of length $l$ involved in one transition, $\left\langle n_{\mathrm{IS}}(l)\right\rangle$. This quantity is related with the average number of particles moving in strings during one IS transition by $\left\langle\mathcal{N}_{\text {IS }}\right\rangle=\Sigma_{l} l\left\langle n_{\text {IS }}(l)\right\rangle$. For $T_{l}$ and $T_{h}$, the decrease of $\left\langle n_{\text {IS }}(l)\right\rangle$ is consistent with an exponential decay. However, we find that the functional form of the decay depends somewhat on the choice of $\delta$. Moreover, finite size effects can be expected for $l \geqslant 5$ due to the small system size. In any case, exponential decays were also observed for the probability distribution of the string length when analyzing the dynamics of equilibrium liquids. ${ }^{17,29,45,46} \mathrm{We}$ emphasize that the observed temperature dependence of $\left\langle\mathcal{N}_{\text {IS }}\right\rangle$ does not imply that string-like motion is more important at higher temperatures. Instead, one must take into account that only particles that show a certain minimum $d r_{\text {IS }}$ can fulfill the above specified 


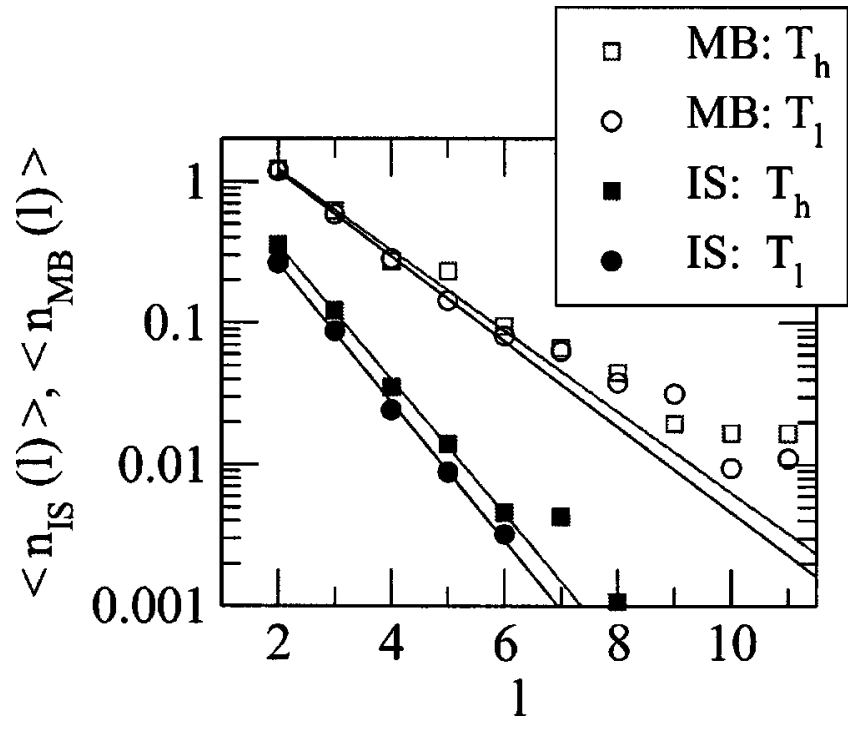

FIG. 6. Mean number of strings of length $l$ observed during transitions between successive inherent structures $\left(\left\langle n_{\mathrm{IS}}(l)\right\rangle\right)$ and metabasins $\left(\left\langle n_{\mathrm{MB}}(l)\right\rangle\right)$, respectively. Data for temperatures $T_{l}=0.435$ and $T_{h}=0.500$ are shown. (Lines) Interpolations with an exponential decay.

replacement criterion and, thus, a larger mean displacement—as was observed at the higher temperature, (cf. Fig. 1) tends to lead to a larger number $\left\langle\mathcal{N}_{\text {IS }}\right\rangle$. Indeed, it was found for equilibrium liquids that the fraction of particles moving in strings increases upon cooling. ${ }^{17,29}$

Finally, we study the localization of particle rearrangements resulting from IS transitions. For this purpose, we measure the number of involved particles by the quantities $z_{1, \mathrm{IS}}$ and $z_{2, \mathrm{IS}}$. The former is defined as

$$
z_{1, \mathrm{IS}}=\sum_{i \in A} \frac{d r_{\mathrm{IS}}^{i}}{d R_{\mathrm{IS}}}
$$

where $d R_{\mathrm{IS}}$ is the maximum displacement of an A particle during the respective transition, and the latter is calculated according to ${ }^{47}$

$$
z_{2, \mathrm{IS}}=\frac{\left[\Sigma_{i \in A}\left(d r_{\mathrm{IS}}^{i}\right)^{2}\right]^{2}}{\sum_{i \in A}\left(d r_{\mathrm{IS}}^{i}\right)^{4}}
$$

In the case where $n$ particles move the same distance and the remainder is immobile, $z_{1, \text { IS }}$ and $z_{2, \text { IS }}$ equal $n$. In Fig. 7, we show the probability distribution $p\left(z_{1, \mathrm{IS}}\right)$ for the studied temperatures. For $T_{l}$ and $T_{h}$, the distributions are nearly symmetric and peak at $z_{1, I S} \approx 16$. The shape of all curves can be described by a Gaussian with a width parameter $\sigma \approx 4.5$, which again reflects the diversity of IS dynamics. The distributions $p\left(z_{2, I S}\right)$ (not shown) are close to a Gaussian centered at $z_{2, \mathrm{IS}} \approx 17$ and characterized by $\sigma \approx 7.5$. A closer inspection reveals that the mean values $\left\langle z_{1, \mathrm{IS}}\right\rangle$ and $\left\langle z_{2, \mathrm{IS}}\right\rangle$ decrease by about 0.5 when decreasing the temperature from $T_{h}$ to $T_{l}$, i.e., IS dynamics becomes slightly more local upon cooling. We emphasize that the value of about 16 for the number of particles involved in IS dynamics should not be taken too literally since measures of the localization different from $z_{1, \mathrm{IS}}$ and $z_{2, \text { IS }}$ can yield values that are up to a factor of 3 smaller.

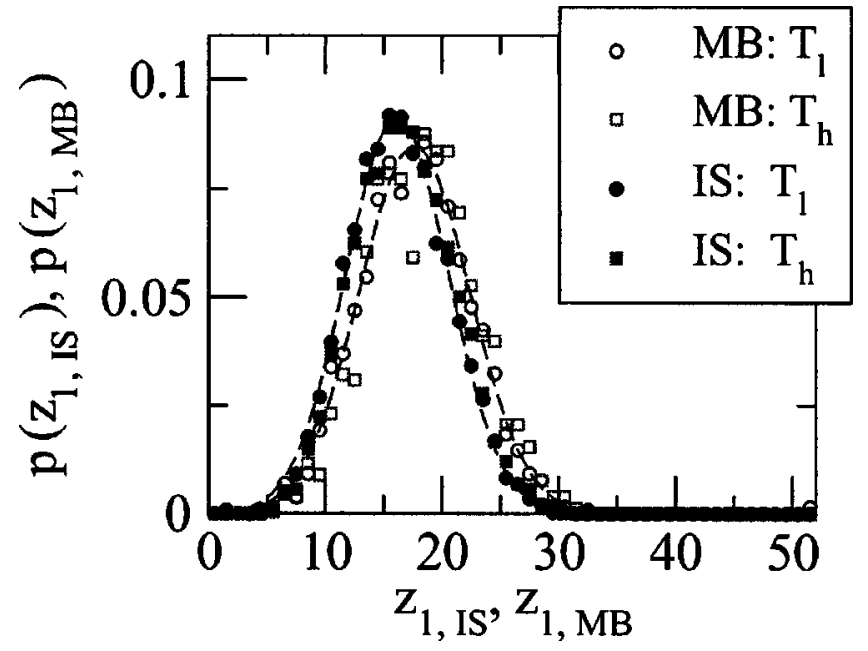

FIG. 7. Probability distributions $p\left(z_{1, \mathrm{IS}}\right)$ and $p\left(z_{1, \mathrm{MB}}\right)$ characterizing the number of particles participating in the rearrangements due to transitions between successive inherent structures and metabasins, respectively [cf. Eq. (2)]. The results for $T_{l}=0.435$ and $T_{h}=0.500$ are compared. (Dashed lines) Interpolations of the data for $T_{l}$ with a Gaussian.

\section{B. Transitions between metabasins}

Recalling that Stillinger ${ }^{3,4}$ related the $\alpha$ process to MB transitions, we now turn to the particle rearrangements during these transitions. For this purpose, we search the IS with the lowest energy in each $\mathrm{MB}, \xi\left(t_{k}\right)$, where $k$ is the index of the $\mathrm{MB}$, and define the particle displacement during a $\mathrm{MB}$ transition as the one obtained by a comparison of $\xi\left(t_{k}\right)$ and $\xi\left(t_{k+1}\right)$. Since $k$ and $k+1$ are not the indices of consecutive IS, but of consecutive MB, these displacements are caused by a sequence of IS transitions. In what follows, we describe the particle displacement during a MB transition by the vector $\mathbf{d r}_{\mathrm{MB}}(k)$ and denote its absolute value as $d r_{\mathrm{MB}}$.

The probability distributions $p\left(d r_{\mathrm{MB}}\right)$ calculated for $T_{l}$ and $T_{h}$ are included in Fig. 1. Since MB dynamics results from several IS transitions, the mean displacement $\left\langle d r_{\mathrm{MB}}(T)\right\rangle$ is larger than $\left\langle d r_{\mathrm{IS}}(T)\right\rangle$. For the temperature dependence of MB dynamics, we find different mean values $\left\langle d r_{\mathrm{MB}}\left(T_{l}\right)\right\rangle=0.18$ and $\left\langle d r_{\mathrm{MB}}\left(T_{h}\right)\right\rangle=0.20$, but the shape of the distributions $p\left(d r_{\mathrm{MB}}\right)$ at $T_{l}$ and $T_{h}$ is comparable. Similar to $p\left(d r_{\mathrm{IS}}\right)$, the distributions are well described by an exponential decay at large $d r_{\mathrm{MB}}$. Interestingly, for neither type of transition, there is enhanced probability at $d r_{\mathrm{IS} / \mathrm{MB}}$ $\approx 1.0$. Hence, single-particle hopping on the length scale of the interparticle distance observed for various larger models of equilibrium liquids at $T \approx T_{\mathrm{MCT}}{ }^{9,15,31,32}$ does not manifest itself in IS and MB dynamics of our small system. The mean energy difference of the IS involved in the MB transition, $\left\langle d E_{\mathrm{MB}}\right\rangle$, increases from 1.42 at $T_{l}$ to 1.56 at $T_{h}$.

To analyze whether particles showing similar mobilities during MB transitions are spatially correlated we calculate the probability distributions $p\left(m_{\mathrm{MB}}\right)$ which, analogous to $p\left(m_{\mathrm{IS}}\right)$, characterize the mobility of the neighbors of the most mobile and the most immobile A particle during a MB transition. It is evident from Fig. 4 that the most mobile particle during a MB transition is prevailingly surrounded by other mobile particles. Moreover, a comparison of $p\left(m_{\mathrm{IS}}\right)$ and $p\left(m_{\mathrm{MB}}\right)$ shows that the spatially heterogeneous nature of 
IS and MB dynamics is very similar at $T \approx T_{\mathrm{MCT}}$. To gain further valuable insights, we study the localization of the particle rearrangements due to $\mathrm{MB}$ transitions using the quantity $z_{1, \mathrm{MB}}$ defined in analogy to Eq. (2). Inspecting the probability distributions $p\left(z_{1, \mathrm{MB}}\right)$ for $T_{l}$ and $T_{h}$ in Fig. 7, we see that a comparable number of particles participates in IS and $\mathrm{MB}$ dynamics, suggesting that basically the same group of particles is "active" during all IS transitions involved in the respective MB transition. Further, similar to IS dynamics, MB dynamics is slightly less local at the higher temperature $\left(\left\langle z_{1, \mathrm{MB}}\left(T_{l}\right)\right\rangle=17.7,\left\langle z_{1, \mathrm{MB}}\left(T_{h}\right)\right\rangle=18.3\right)$. Hence, we find no indication that the length scales attributed to the spatially heterogeneous nature of IS and MB dynamics, respectively, increase upon cooling.

We further explore the spatial heterogeneities associated with MB dynamics by investigating the relevance of stringlike motion. With the same definition as in the previous section, we find that string-like motion occurs during $87 \%$ of all MB transitions at $T_{l}$ where the average number of particles that move in strings during a MB transition amounts to $\left\langle\mathcal{N}_{\mathrm{MB}}\right\rangle=7.2$. These numbers indicate that string-like motion yields an important contribution to the particle rearrangements during $\mathrm{MB}$ transitions. In other words, the group of particles that takes part in $\mathrm{MB}$ dynamics usually achieves the large displacements by means of string-like motion. The relevance of this type of motion for MB dynamics is also obvious from Fig. 6 where we display the distributions $\left\langle n_{\mathrm{MB}}(l)\right\rangle$ characterizing the mean number of strings of length $l$ during one MB transition.

\section{Sequences of inherent structure transitions}

Due to the organization of the IS into MB, two different processes are relevant when the system samples the PEL. First, the exploration of a MB, i.e., the back-and-forth jumps between the IS in the same MB and, second, the transition between distinct MB. In this section, we study the particle rearrangements involved in these processes by following the particle rearrangements during suitable sequences of IS transitions. Since the diffusion constant is basically determined by the trapping of the system in long-lived $\mathrm{MB},{ }^{24,25}$ we now focus on MB within which at least six IS transitions occur, i.e., we consider $49 \%$ of all MB. To investigate the exploration process and the transition process separately we construct $E$ and $T$ sequences using the following criteria: An $E$ sequence combines all IS transitions connecting the two IS within the same MB that show the largest distance $d r_{\mathrm{IS}}^{C}$. This means that, on average, nine IS transitions form an $E$ sequence for $T_{l}$. To obtain the $T$ sequences we unite four IS transitions that occur when the system moves to another MB. More precisely, if two successive MB are separated by more than four IS transitions, we choose four jumps in the middle of this series. On the other hand, if the MB are separated by less than four IS transitions, we add the adjoining transitions within the involved $\mathrm{MB}$ to the sequence. We do not regard the latter procedure as a serious problem because, in any case, long-lived MB are exited by a series of events ${ }^{24}$ so that it is not completely clear which individual transitions are a part of the escape process. The chosen numbers are also

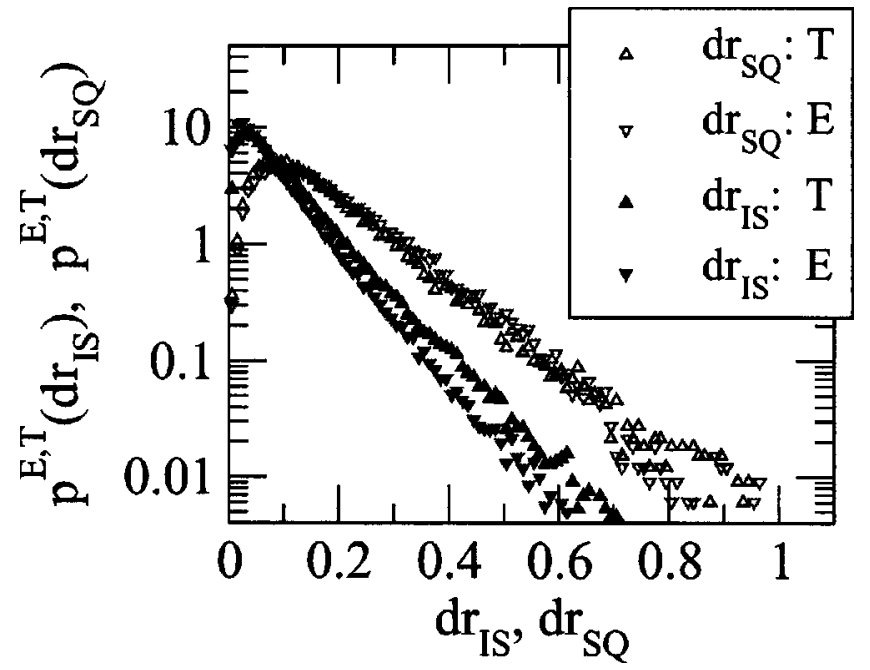

FIG. 8. Probability distributions $p^{E}\left(d r_{\mathrm{SQ}}\right)$ and $p^{T}\left(d r_{\mathrm{SQ}}\right)$ describing the displacements of the A particles during sequences of inherent structure transitions that occur when a metabasin is explored $(E)$ and when a transition between different meta basins takes place $(T)$, respectively $(T=0.435)$. For comparison, we included the corresponding results for the involved individual transitions, $p^{E}\left(d r_{\mathrm{IS}}\right)$ and $p^{T}\left(d r_{\mathrm{IS}}\right)$.

motivated by the overall displacements during the $E$ and $T$ sequences, see below. However, we ensured that the basic features of our results are unchanged when the numbers are varied in a meaningful range. The IS transitions involved in the $E$ and $T$ sequences, respectively, are denoted as $E$ and $T$ transitions in what follows.

Figure 8 shows the probability distributions $p^{E, T}\left(d r_{\mathrm{SQ}}\right)$ and $p^{E, T}\left(d r_{\mathrm{IS}}\right)$ for the displacements of the A particles during the $E / T$ sequences and the $E / T$ transitions at $T_{l}$, respectively. While different mean displacements $\left\langle d r_{\mathrm{IS}}\right\rangle^{T}$ $>\left\langle d r_{\mathrm{IS}}\right\rangle^{E}$ are found for the single transitions, the rearrangements during the $E$ and the $T$ sequences are described by nearly identical distribution functions. Thus, the smaller mean displacements during the $E$ transitions and the backand-forth jumps between IS within the same MB lead to the effect that four $T$ transitions result in the same overall displacement as, on average, nine $E$ transitions. In other words, the particle rearrangements during the exploration and the transition process are very similar at the level of sequences of transitions. Despite the quantitative differences, the distributions for the single transitions, $p^{E}\left(d r_{\text {IS }}\right)$ and $p^{T}\left(d r_{\text {IS }}\right)$ are still comparable. In particular, due to the correlation between the particle displacement and the energy difference $d E_{\mathrm{IS}}$, (cf. Fig. 2) some of the deviations result from the fact that larger mean energy differences are found for the $T$ transitions. Strictly speaking, when calculating the distributions $p^{E}\left(d r_{\mathrm{IS}} ; d E_{\mathrm{IS}}\right)$ and $p^{T}\left(d r_{\mathrm{IS}} ; d E_{\mathrm{IS}}\right)$ we find that both distributions are very similar for IS transitions characterized by high energy differences, but somewhat different in the case of small $d E_{\mathrm{IS}}$.

The finding $p^{E}\left(d r_{\mathrm{SQ}}\right) \approx p^{T}\left(d r_{\mathrm{SQ}}\right)$ enables us to study the relevance of string-like motion for the exploration and the transition process without any effect of the respective mean particle displacement (see the discussion of Fig. 6). This was one reason for the specific choice of the parameters when constructing the sequences. The distributions $\left\langle n_{\mathrm{SQ}}(l)\right\rangle^{E, T}$ for 


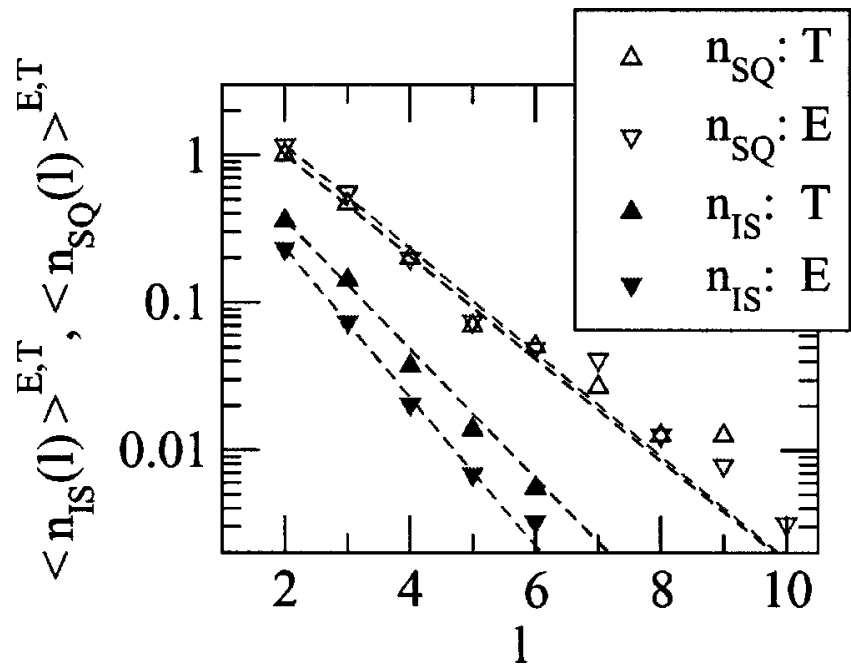

FIG. 9. Mean number of strings of length $l$ resulting from: (i) single transitions between successive inherent structures visited during the exploration $\left(\left\langle n_{\mathrm{IS}}(l)\right\rangle^{E}\right)$ and the transition process $\left(\left\langle n_{\mathrm{IS}}(l)\right\rangle^{T}\right)$ and (ii) sequences of transitions of inherent structures visited during the exploration $\left(\left\langle n_{\mathrm{SQ}}(l)\right\rangle^{E}\right)$ and the transition process $\left(\left\langle n_{\mathrm{SQ}}(l)\right\rangle^{T}\right)(T=0.435)$. (Lines) Interpolations with an exponential decay.

the mean number of strings of length $l$ during one $E$ and one $T$ sequence at $T_{l}$, respectively, are displayed in Fig. 9. The good agreement indicates that string-like motion is of similar importance for the particle rearrangements during the exploration and the transition process, respectively. This finding excludes that it is simply the occurrence of string-like motion that allows the system to escape from a MB. This point will be further discussed later in this section. For comparison, we include the distributions $\left\langle n_{\mathrm{IS}}(l)\right\rangle^{E, T}$ characterizing string-like motion during the individual $E$ and $T$ transitions, in Fig. 9. Both distributions can be interpolated by an exponential decay, suggesting that the basic features of string-like motion are comparable for the different types of transitions. As was discussed above, the quantitative differences result at least in part from the distinct mean particle displacements due to the $E$ and the $T$ transitions (cf. Fig. 8). Taking also into account that a comparable number of particle participates in $E$ and $T$ transitions $\left(\left\langle z_{1, \mathrm{IS}}\right\rangle^{E}=16.3,\left\langle z_{1, \mathrm{IS}}\right\rangle^{T}=16.3\right.$ for $\left.T_{l}\right)$ we conclude that the different types of transitions show at most some quantitative differences in the vicinity of $T_{\mathrm{MCT}}$, supporting our prior finding that IS dynamics in different regions of the PEL are comparable (see Fig. 3).

Next, we study the mechanism of string-like motion. When comparing $\left\langle n_{\mathrm{SQ}}(l)\right\rangle^{E, T}$ with their counterparts for the single transitions $\left\langle n_{\mathrm{IS}}(l)\right\rangle^{E, T}$ (cf. Fig. 9), it becomes obvious that the distributions have different slopes in a semilogarithmic representation. Hence, one may speculate that a string observed during a sequence does not result from a single transition, but from the interplay of particle displacements due to subsequent jumps. For example, one can imagine that a long "macrostring," defined as a string during a sequence, is composed of several "microstrings" resulting from the displacements during the individual transitions of the series. This will now be studied in more detail. For this purpose, we consider all macrostrings of length $l \geqslant 3$ and analyze how many of them result from a single transition. We find that only $15.4 \%$ and $13.7 \%$ of the macrostrings during the $E$ and the $T$ sequences, respectively, are the consequence of a single event. On the other hand, $48.4 \%(E)$ and $57.5 \%(T)$ of the macrostrings are composed of at least one microstring. The remainder, namely, $36.2 \%(E)$ and $28.8 \%(T)$, can be traced back to concerted single-particle type displacements in successive jumps. Likewise, for $47.8 \%(E)$ and $53.8 \%(T)$ of all particles involved in macrostrings of length $l \geqslant 3$, the replacement of the neighboring particle takes place during a single transition, while, for the remainder of the particles, several displacements must add up for the replacement criterion to be fulfilled. Of course, these numbers depend on the definition of the strings. Nevertheless, they show that most macrostrings, especially, the long ones, do not result from a coherent motion of all particles, but from subsequent motions of single particles or small groups of particles.

A typical example of the interplay of microstrings and single-particle motions in forming a large macrostring is shown in Fig. 5, where the different shades of gray denote particles replacing each other at different times. It is also seen that the replacements do not start and end at the "head" (right hand site) and the "tail" (left hand side) of the string, respectively, but occur in a random order. Though ordered sequential replacements along the string are observed in many instances, we still regard the scenario in Fig. 5 as a typical example.

Especially, the macrostrings of the $E$ sequences may be a subtle result of individual motions. Since the IS within a MB are visited several times, the microstrings of the $E$ sequences may show back-and-forth jumps. To quantify this effect we calculated the probability that a microstring observed during an $E$ transition jumps back during a later transition in the same MB. We find that for $44.5 \%$ of the microstrings all particles jump back to their initial positions. Hence, backand-forth jumps of microstrings are a frequent phenomenon when observing IS dynamics within a MB. In comparison, the back-jump probability for the microstrings during the $T$ sequences amounts to only $2 \%$. Considering also the results in Fig. 9, one may speculate that, though string-like motion is of similar relevance for the particle rearrangements during the exploration and the transition process, respectively, it is the occurrence of successful strings that enables the system to escape from a MB.

\section{Correlation of successive particle displacements}

The results of Doliwa and Heuer ${ }^{24,25}$ suggest that jumps between MB resemble a random walk on the PEL. To check this conclusion, we study the correlation of particle displacements resulting from MB transitions at two different times, i.e., four-time correlation functions are considered. To measure for how many MB transitions an A particle remembers the direction of an initial motion we define the correlation function

$$
D(\Delta k)=\left\langle\frac{\mathbf{d} \mathbf{r}_{\mathrm{MB}}(k)}{\mathbf{d \mathbf { r } _ { \mathrm { MB } } ( k )}} \cdot \frac{\mathbf{d \mathbf { r } _ { \mathrm { MB } } ( k + \Delta k )}}{\mathbf{d \mathbf { r } _ { \mathrm { MB } } ( k + \Delta k )}}\right\rangle .
$$

Here, the brackets $\langle\ldots\rangle$ denote the average over all MB transitions and all A particles. Due to the properties of the scalar 

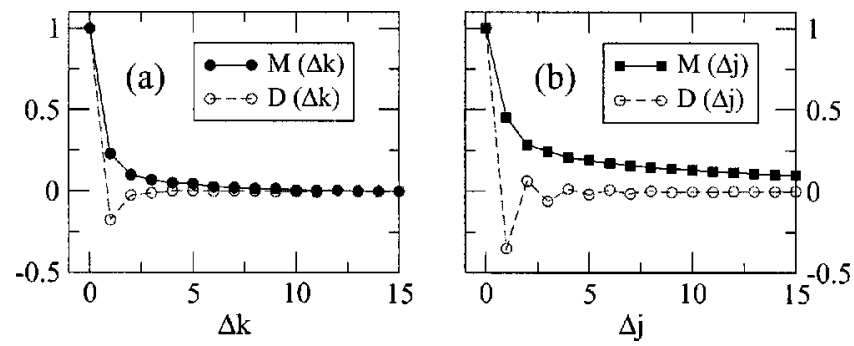

FIG. 10. (a) Correlation functions that relate the displacements of single A particles during different transitions between metabasins at $T=0.435$. $D(\Delta k)$ measures the correlation of the directions of subsequent motions; $M(\Delta k)$ correlates the respective relative mobilities, [cf. Eqs. (4) and (6)]. (b) Analogously defined quantities $D(\Delta j)$ and $M(\Delta j)$ characterizing the correlation of the particle displacements during different transitions between inherent structures.

product, $D(\Delta k)$ will be positive (negative) if, on average, the displacements of a particle during the MB transitions $\xi\left(t_{k}\right) \rightarrow \xi\left(t_{k+1}\right)$ and $\xi\left(t_{k+\Delta k}\right) \rightarrow \xi\left(t_{k+\Delta k+1}\right)$, respectively, have the same (opposite) direction. Another property of the particle displacement during a MB transition is the mobility. Different from the definition used so far, we now characterize the relative mobility of a particle by

$$
\mu(k)=\frac{d r_{\mathrm{MB}}(k)-\left\langle d r_{\mathrm{MB}}(k)\right\rangle}{\left\langle d r_{\mathrm{MB}}(k)\right\rangle},
$$

where $\left\langle d r_{\mathrm{MB}}(k)\right\rangle$ is the mean displacement of an A particle during the MB transition $\xi\left(t_{k}\right) \rightarrow \xi\left(t_{k+1}\right)$. Hence, the correlation function

$$
M(\Delta k)=\langle\mu(k) \mu(k+\Delta k)\rangle
$$

relates the relative mobilities during different MB transitions. To study the correlation of the particle displacements resulting from different IS transitions, we define the correlation functions $D(\Delta j)$ and $M(\Delta j)$ in analogy to their counterparts for the MB transitions.

Figure 10(a) shows $D(\Delta k)$ and $M(\Delta k)$ for the MB dynamics at $T_{l}$. Inspecting the latter correlation function a rapid decay is evident. For the former, the negative sign of $D(\Delta k=-1)$ implies that subsequent motions are backward correlated, i.e., the particles tend to move towards their old positions during the next jump. However, the values $\mid D(\Delta k$ $=1) \mid \ll 1$ and $M(\Delta k=1) \ll 1$ indicate that the particle rearrangements during consecutive MB transitions show only a small correlation. Hence, a random walk on the PEL is indeed a good approximation for the jumps between MB. In addition, the findings imply that, during different MB transitions, different groups of particles are highly mobile and form strings, corroborating our speculation that the occurrence of a successful string results in the escape from a MB.

For comparison, $D(\Delta j)$ and $M(\Delta j)$ characterizing IS dynamics at $T_{l}$ are displayed in Fig. 10(b). Due to the backand-forth jumps between the IS within a MB the correlations persist for several IS transitions. In particular, sequences of back-and-forth jumps between two IS result in a oscillatory behavior of $D(\Delta j) . M(\Delta j)$ does not completely decay within the first 15 IS transitions. Since the same transition may occur several times when the system explores a $\mathrm{MB}$, the information about the initial mobility is not completely lost until the MB is exited.

Very recently, Garrahan and Chandler ${ }^{48,49}$ proposed that bulk dynamics in supercooled liquids can be understood based on two simple ingredients, namely, the existence of spatially heterogeneous dynamics and the facilitation of dynamics in the vicinity of mobile regions. To check this assumption, we study whether strings during consecutive MB transitions are spatially correlated. For this purpose, we first characterize the positions of all strings by their center of mass. Then, for every string during the MB transition $k \rightarrow k$ +1 , the minimum distance between its position and the positions of any string during the transition $k-1 \rightarrow k$ is determined. Finally, we repeat this calculation for randomly chosen positions of the strings during the MB transition $k \rightarrow k$ +1 . For the actual strings at $T_{l}$, we obtain a mean minimum distance $\langle d\rangle=1.3$ which is slightly smaller than $\langle d\rangle=1.5$ found for a random distribution, suggesting that dynamics in the vicinity of mobile regions may be somewhat facilitated. However, the observed effect is weak and needs to be validated for larger systems.

\section{DISCUSSION AND SUMMARY}

We studied the particle rearrangements during transitions between consecutive IS and consecutive MBs of a 80:20 BLJ liquid at $T_{h}>T_{\mathrm{MCT}}$ and $T_{l} \leqslant T_{\mathrm{MCT}}$. Considering that the motion of the system on the PEL can be decomposed into the exploration of $\mathrm{MB}$ and the transition between distinct $\mathrm{MB}$, we compared the particle rearrangements resulting from both processes. This analysis was done both at the level of single transitions ( $E$ and $T$ transitions) and at the level of suitable sequences of transitions ( $E$ and $T$ sequences). Since the time constants for the dynamics of the A and the B particles of the BLJ mixture are somewhat different, ${ }^{33,34}$ we mostly focused on the motion of the former. However, with respect to all studied quantities, only minor differences are observed for the B particles.

Comparing the results for $T_{h}>T_{\mathrm{MCT}}$ and $T_{l} \leqslant T_{\mathrm{MCT}}$, no change of the basic features except only gradual variations were found. For IS and MB dynamics, the mean energy differences $\left\langle d E_{\mathrm{IS} / \mathrm{MB}}\right\rangle$, the mean displacement of the A particles $\left\langle d r_{\mathrm{IS} / \mathrm{MB}}\right\rangle$, and the mean number of A particles taking part in a transition $\left\langle z_{1, \mathrm{IS} / \mathrm{MB}}\right\rangle$, decrease upon cooling, but the shape of the corresponding distributions is basically unchanged. For $T_{l}$ and $T_{h}$, the distributions $p\left(z_{1, \mathrm{IS} / \mathrm{MB}}\right)$ are close to Gaussian and, over a wide range, the curves $p\left(d r_{\mathrm{IS} / \mathrm{MB}}\right)$ decay exponentially. The decrease of $\left\langle d E_{\mathrm{IS} / \mathrm{MB}}\right\rangle$ is consistent with the decline of $k_{B} T$. The variation of $\left\langle d r_{\mathrm{IS} / \mathrm{MB}}\right\rangle$ is, at least in part, a consequence of the changing mean energy difference, because it was found that large changes of the energy are accompanied by large particle displacements. Hence, when observing IS and MB dynamics in a 65-particle BLJ liquid, there is no evidence that the mechanism for the particle motion is discontinuously altered at $T_{\mathrm{MCT}}$. In particular, we do not find single-particle hopping on the length scale of the interparticle distance.

These results are consistent with findings by Schrøder et al. ${ }^{15}$ who concluded that single-particle hopping observed 
for the equilibrium liquid at $T \approx T_{\mathrm{MCT}}$ does not result from transitions over single barriers, but "the jump occurs via a number of intermediate IS." At variance with the outcome of the present work, Hernandez-Rojas and Wales ${ }^{27}$ reported a "distinct change in behavior" at $T \approx T_{\mathrm{MCT}}$ when investigating the particle rearrangements in a kinetic Monte Carlo approach. Since $T_{l}=0.435$ is still close to $T_{\mathrm{MCT}}=0.45 \pm 0.01$ it may be useful to check our results over a broader temperature range. With this reservation, the absence of a significant change of the dynamical behavior at $T_{\mathrm{MCT}}$ supports that the sampling of the PEL changes gradually ${ }^{24,26}$ instead of discontinuously as concluded in normal-mode analysis approaches. ${ }^{18-22}$ On the other hand, since our findings are related to the exploration of the PEL they do not allow us to draw conclusions about the validity of MCT predictions for the equilibrium liquid.

In addition, we analyzed the dependence of IS dynamics on the energy of the initial IS, $E_{\text {IS }}$. Weak variations of $\left\langle d r_{\mathrm{IS}}\left(E_{\mathrm{IS}}\right)\right\rangle$ indicate that the energy has a minor influence. These results suggest that IS dynamics in different regions of the PEL are comparable. Such behavior is in accordance with the current picture of the PEL of fragile glass formers where a comparable ruggedness is assumed throughout the landscape. ${ }^{3,4}$ Moreover, a weak dependence of IS dynamics on $E_{\text {IS }}$ is consistent with our findings for the particle rearrangements during $E$ and $T$ transitions, i.e., for IS dynamics occurring when a MB is explored and when a transition between different MB takes place, respectively. We observed that the mean displacement $\left\langle d r_{\mathrm{IS}}\right\rangle^{T}$, is somewhat larger than $\left\langle d r_{\mathrm{IS}}\right\rangle^{E}$, but this effect results in large parts from larger mean energy differences during the $T$ transitions. In addition, the number of particles involved in IS dynamics and in stringlike motion are similar during the exploration and the transition process. Hence, with respect to the accompanying particle rearrangements, no principal differences exist between IS transitions near the bottom of a MB and near the ridges between distinct MB.

We further showed that the particles participating in IS and MB dynamics, respectively, i.e., particles showing high displacements are not randomly distributed, but rather reside prevailingly in the next neighbor shell of each other. Thus, consistent with previous computational work on supercooled liquids, ${ }^{17,29,30,43,44}$ IS and MB dynamics at $T \approx T_{\mathrm{MCT}}$ are spatially heterogeneous. In experiments, ${ }^{40,41}$ a spatially heterogeneous nature was observed for dynamics near $T_{g}$. Though the dynamical heterogeneities at $T \approx T_{\mathrm{MCT}}$ and at $T \geqslant T_{g}$, respectively, show similar features, e.g., a comparable rate memory, ${ }^{50}$ their exact relation is still elusive.

An analysis of string-like motion revealed that this dynamical pattern is observed during $29 \%$ of the IS transitions and during $87 \%$ of the MB transitions at $T_{l}$. The latter value together with $\left\langle\mathcal{N}_{\mathrm{MB}}\right\rangle=7.2$ obtained for the mean number of particles forming strings per MB transition indicate that string-like motion is very important for MB dynamics. On the other hand, this type of motion appears to be less relevant on the shorter time scale of IS dynamics. To gain further insights, we compared string-like motion during sequences of IS transitions that take place when the system explores a $\mathrm{MB}$ and when it moves from one MB to another, respec- tively. Constructing $E$ and $T$ sequences such that comparable particle displacements result, we observed $\left\langle n_{\mathrm{SQ}}(l)\right\rangle^{E}$ $\approx\left\langle n_{\mathrm{SQ}}(l)\right\rangle^{T}$ for the mean number of strings of length $l$ during the respective sequences. Thus, string-like motion during the exploration and the transition process is not different, suggesting that the probability to find a string depends merely on the displacements of the particles.

Concerning the mechanism of string-like motion, it was demonstrated that the formation of strings results from the concerted interplay of the particle rearrangements related to subsequent IS transitions. In other words, most macrostrings defined as strings that result from a series of transitions do not arise from a coherent motion of all particles at the same time, but from coordinated displacements of subunits at different times. These subunits include both single particles and microstrings, i.e., small groups of particles replacing each other within one transition. Further, the subsequent particle replacements in a macrostring do not necessarily start at the "head" of the string and end at the "tail", but successive replacements often take place at random positions in the string (cf. Fig. 5). For IS dynamics inside the MB, the formation of a macrostring frequently includes back-and-forth jumps of the involved particles. All these findings elucidate that string-like motion is a complex dynamical process in which several rearrangements within a group of particles occur. In view of this result, the observation $\left\langle z_{1, \mathrm{IS}}\right\rangle \approx\left\langle z_{1, \mathrm{MB}}\right\rangle$ for the number of particles taking part in IS and MB dynamics, respectively, becomes plausible. Since particles that are "active" during a MB transition mostly participate in strings, the multistep nature of this type of motion has the consequence that basically the same group of particles is active during all IS transitions involved in the respective MB transition.

The multistep nature of string-like motion appears to support Garrahan and Chandler ${ }^{48,49}$ who stress the importance of dynamic facilitation, i.e., they assume that regions showing high mobility assist neighboring regions to become mobile. However, the formation of strings may also be related to local structural properties of supercooled liquids. Based on the mechanism of string-like motion Gebremichael et al. ${ }^{46}$ suggest that temporary fissures open a quasi-onedimensional channel in which the particles can move in strings. Further investigation on this point is underway. ${ }^{51}$

Finally, we studied the correlation of particle displacements during different transitions between MB, i.e., time correlation functions of displacements were investigated. We find that the displacements of any one particular particle during consecutive MB transitions are basically uncorrelated. In other words, different groups of particles are mobile during successive MB transitions. In contrast, when relating the particle rearrangements due to different IS transitions, the backand-forth jumps between the IS within a MB and the process of string formation result in a correlation of the particle displacements that persists for several IS transitions. To check the concept of dynamic facilitation at the level of MB dynamics we investigated whether the strings during two consecutive MB transitions are spatially correlated. For this purpose, we calculated the minimum distance between a new string and any of the old strings. We found a mean minimum distance that is $15 \%$ smaller than in the case of a random 
distribution of the new strings, suggesting that new strings tend to be formed near regions where string-like motion has taken place. This finding is again consistent with the concept of dynamic facilitation. However, the effects observed for MB dynamics are weak and need to be validated in future investigations.

Stillinger ${ }^{3,4}$ assigned the $\alpha$ and the (Johari-Goldstein) $\beta$ process $^{28}$ to MB and IS dynamics, respectively. From experimental work, it is known that both processes often merge into a single high temperature relaxation near $T_{\mathrm{MCT}} \cdot{ }^{28,52,53}$ Multidimensional nuclear magnetic resonance experiments have shown that the molecular reorientations associated with the $\alpha^{54-56}$ and the $\beta$ process $^{57-59}$ close to $T_{g}$ are complex multistep processes. The multistep reorientation during the $\beta$ relaxation is restricted to a small section of the unit sphere $^{57-60}$ where the accessible solid angle grows with increasing temperature. ${ }^{28,53,58}$ In addition, computational studies have revealed that the length scale associated with the spatially heterogeneous nature of the $\alpha$ process increases upon cooling $T \rightarrow T_{\mathrm{MCT}}{ }^{29,61-63}$

Several of the present and the summarized results are inconsistent with the assumption that the $\alpha$ process is identical with MB dynamics. First, we found that MB dynamics results in a mean particle displacement that is much smaller than the interparticle distance, indicating that, at the studied temperatures, the structural relaxation is not complete after a single MB transition. Likewise, $\tau_{\alpha}$ is approximately a factor of 30 longer than the mean waiting time in the MB $\left\langle\tau_{\mathrm{MB}}\right\rangle{ }^{24,25}$ Finally, the temperature dependence of the mean number of particles involved in a MB transition, $\left\langle z_{1, \mathrm{MB}}\right\rangle$, is incompatible with an increasing length scale attributed to the spatial heterogeneities of MB dynamics. Concerning the $\beta$ process, some of the experimental results appear to be at variance with the assumption that this relaxation is related to single transitions between IS. Specifically, the experimental finding that the $\beta$ process results from a multistep process is inconsistent with a single jump in the PEL. Moreover, the temperature dependent $\beta$ relaxation strength is difficult to understand. In particular, an explanation is problematic when IS dynamics in different regions are similar, as suggested by our results.

In view of these findings, we propose that the MB transitions are the elementary steps of the $\alpha$ process or, equivalently, the structural relaxation results from a series of $\mathrm{MB}$ transitions. Further, we suggest that the $\beta$ process is related to the exploration of a $\mathrm{MB}$, i.e., to the $E$ sequences. In this picture, both relaxation phenomena are naturally multistep processes. Further, the growing time scale separation of the $\alpha$ and the $\beta$ process upon cooling is a consequence of the increasingly important trapping of the system in a MB. When decreasing the temperature the system is forced into deeper and deeper regions of the $\mathrm{MB}$ so that smaller and smaller regions of the $\mathrm{MB}$ can be explored due to the $\beta$ process and, consequently, its relaxation strength decreases. In other words, the number of IS visited during the $\beta$ process is temperature dependent. Of course, our speculations need to be carefully checked in future investigations.

In conclusion, the following picture appears to emerge for IS and MB dynamics at sufficiently low temperatures.
When the system jumps between IS organized into a MB, groups of spatially correlated particles achieve comparatively large displacements by performing string-like motion. In doing so, the strings arise, for the most part, not due to a coherent motion of all involved particles during a single IS transition, but from the interplay of displacements of various subunits taking place at different times. During the exploration of the MB, the system enters energetically less favorable regions near the ridge of the $\mathrm{MB}$ from which it either escapes to a new MB or returns to the bottom of the old MB. While in the latter case, the formed strings dissolve due to the backward motions of the involved particles, they persist in the former. The particle rearrangements during different $\mathrm{MB}$ transitions are basically uncorrelated. In particular, different groups of particles are mobile and form strings. Hence, one may speculate that successful string-like motion results in the escape from a MB.

\section{ACKNOWLEDGMENTS}

The authors thank Y. Gebremichael for stimulating discussions. M.V. gratefully acknowledges funding by the Deutsche Forschungsgemeinschaft (DFG) through the EmmyNoether Programm.

${ }^{1}$ M. Goldstein, J. Chem. Phys. 51, 3728 (1969).

${ }^{2}$ C. A. Angell, Science 267, 1924 (1995).

${ }^{3}$ F. H. Stillinger, Science 267, 1935 (1995).

${ }^{4}$ P. G. Debenedetti and F. H. Stillinger, Nature (London) 410, 259 (2001).

${ }^{5}$ F. H. Stillinger and T. A. Weber, Phys. Rev. A 25, 978 (1982).

${ }^{6}$ F. Sciortino, W. Kob, and P. Tartaglia, Phys. Rev. Lett. 83, 3214 (1999).

${ }^{7}$ S. Büchner and A. Heuer, Phys. Rev. E 60, 6507 (1999).

${ }^{8}$ G. Adam and J. H. Gibbs, J. Chem. Phys. 43, 139 (1965).

${ }^{9}$ S. Sastry, P. G. Debenedetti, and F. H. Stillinger, Nature (London) 393, 554 (1998).

${ }^{10}$ A. Scala, F. W. Starr, E. La Nave, F. Sciortino, and H. E. Stanley, Nature (London) 406, 166 (2000).

${ }^{11}$ S. Sastry, Nature (London) 409, 164 (2001).

${ }^{12}$ I. Saika-Voivod, P. H. Poole, and F. Sciortino, Nature (London) 412, 514 (2001).

${ }^{13}$ J. D. Bryngelson, J. N. Onuchic, N. D. Onuchic, N. D. Socci, and P. G. Wolynes, Proteins 21, 167 (1995).

${ }^{14}$ D. J. Wales, J. P. K. Doye, M. A. Miller, P. N. Mortenson, and T. R. Walsh, Adv. Chem. Phys. 115, 1 (2000).

${ }^{15}$ T. B. Schrøder, S. Sastry, J. C. Dyre, and S. C. Glotzer, J. Chem. Phys. 112, 9834 (2000).

${ }^{16}$ W. Götze and L. Sjogren, Rep. Prog. Phys. 55, 241 (1992).

${ }^{17}$ C. Donati, J. F. Douglas, W. Kob, S. J. Plimpton, P. H. Poole, and S. C. Glotzer, Phys. Rev. Lett. 80, 2338 (1998).

${ }^{18}$ C. Donati, F. Sciortino, and P. Tartaglia, Phys. Rev. Lett. 85, 1464 (2000).

${ }^{19}$ K. Broderix, K. K. Bhattacharya, A. Cavagna, A. Zippeliu, and I. Giardina, Phys. Rev. Lett. 85, 5360 (2000).

${ }^{20}$ L. Angelani, R. Di Leonardo, G. Ruocco, A. Scala, and F. Sciortino, Phys. Rev. Lett. 85, 5356 (2000).

${ }^{21}$ E. La Nave, A. Scala, F. W. Starr, H. E. Staneley, and F. Sciortino, Phys. Rev. E 64, 036102 (2001).

${ }^{22}$ E. La Nave, H. E. Stanley, and F. Sciortino, Phys. Rev. Lett. 88, 035501 (2002).

${ }^{23}$ J. P. K. Doye and D. J. Wales, J. Chem. Phys. 116, 3777 (2002).

${ }^{24}$ B. Doliwa and A. Heuer, Phys. Rev. E 67, 031506 (2003).

${ }^{25}$ B. Doliwa and A. Heuer, Phys. Rev. E 67, 030501 (2003).

${ }^{26}$ R. Aldrin Denny, D. R. Reichman, and J. P. Bouchaud, Phys. Rev. Lett. 90, 025503 (2003).

${ }^{27}$ J. Hernandez-Rojas and D. J. Wales, cond-mat/011212.

${ }^{28}$ G. P. Johari and M. Goldstein, J. Chem. Phys. 53, 2372 (1970).

${ }^{29}$ S. C. Glotzer, J. Non-Cryst. Solids 274, 342 (2000).

${ }^{30}$ H. R. Schober, J. Non-Cryst. Solids 307-310, 40 (2002). 
${ }^{31}$ J.-N. Roux, J.-L. Barrat, and J.-P. Hansen, J. Phys.: Condens. Matter 1, 7171 (1989).

${ }^{32}$ G. Wahnström, Phys. Rev. A 44, 3752 (1991).

${ }^{33}$ W. Kob and H. C. Andersen, Phys. Rev. E 51, 4626 (1995).

${ }^{34}$ W. Kob and H. C. Andersen, Phys. Rev. E 52, 4134 (1995).

${ }^{35}$ S. Büchner and A. Heuer, Phys. Rev. Lett. 84, 2168 (2000).

${ }^{36}$ T. Keyes and J. Chowdhary, Phys. Rev. E 64, 032201 (2001).

${ }^{37}$ T. S. Grigera, A. Cavagna, I. Giardina, and G. Parisi, Phys. Rev. Lett. 88, 055502 (2002).

${ }^{38}$ B. Doliwa and A. Heuer, J. Phys.: Condens. Matter 15, S849 (2003).

${ }^{39}$ B. Doliwa, PhD thesis, University of Mainz, Germany, 2002.

${ }^{40}$ U. Tracht, M. Wilhelm, A. Heuer, H. Feng, K. Schmidt-Rohr, and H. W. Spiess, Phys. Rev. Lett. 81, 2727 (1998).

${ }^{41}$ S. A. Reinsberg, X. H. Qiu, M. Wilhelm, H. W. Spiess, and M. D. Ediger, J. Chem. Phys. 114, 7299 (2001).

${ }^{42}$ H. Sillescu, J. Non-Cryst. Solids 243, 81 (1999), and references therein.

${ }^{43}$ B. Doliwa and A. Heuer, Phys. Rev. Lett. 80, 4915 (1998).

${ }^{44}$ Y. Gebremichael, T. B. Schrøder, F. W. Starr, and S. C. Glotzer, Phys. Rev. E 64, 051503 (2001).

${ }^{45}$ M. Aichele, Y. Gebremichael, F. W. Starr, J. Baschnagel, and S. C. Glotzer, J. Chem. Phys. 119, 5290 (2003).

${ }^{46}$ Y. Gebremichael, M. Vogel, and S. C. Glotzer, J. Chem. Phys. (in press).

${ }^{47}$ F. H. Stillinger and T. A. Weber, Phys. Rev. A 28, 2408 (1983).
${ }^{48}$ J. P. Garrahan and D. Chandler, Phys. Rev. Lett. 89, 035704 (2002).

${ }^{49}$ L. Berthier and J. P. Garrahan, J. Chem. Phys. 119, 4367 (2003).

${ }^{50}$ A. Heuer and K. Okun, J. Chem. Phys. 106, 6176 (1997).

${ }^{51}$ M. Bergroth, A. S. Keyes, M. Vogel, Y. Gebremichael, and S. C. Glotzer (unpublished results).

${ }^{52}$ E. Rössler, Phys. Rev. Lett. 65, 1595 (1990).

${ }^{53}$ A. Kudlik, S. Benkhof, T. Blochowicz, C. Tschirwitz, and E. Rössler, J. Mol. Struct. 479, 201 (1999).

${ }^{54}$ K. Schmidt-Rohr and H. W. Spiess, Multidimensional Solid-State NMR and Polymers (Academic, London, 1994).

${ }^{55}$ R. Böhmer, G. Hinze, G. Diezemann, and E. Rössler, Prog. Nucl. Magn. Reson. Spectrosc. 39, 191 (2001).

${ }^{56}$ U. Tracht, A. Heuer, and H. W. Spiess, J. Chem. Phys. 111, 3720 (1999).

${ }^{57}$ M. Vogel and E. Rössler, J. Phys. Chem. B 104, 4285 (2000).

${ }^{58}$ M. Vogel and E. Rössler, J. Chem. Phys. 114, 5802 (2001).

${ }^{59}$ M. Vogel, C. Tschirwitz, G. Schneider, C. Koplin, P. Medick, and E. Rössler, J. Non-Cryst. Solids 307-310, 326 (2002).

${ }^{60}$ G. Hinze and H. Sillescu, J. Chem. Phys. 104, 314 (1996).

${ }^{61}$ G. Johnson, A. I. Mel'cuk, H. Gould, W. Klein, and R. D. Mountain, Phys. Rev. E 57, 5707 (1998).

${ }^{62}$ R. Ahluwalia and S. P. Das, Phys. Rev. E 57, 5771 (1998).

${ }^{63}$ N. Lacevic, F. W. Starr, T. B. Schrøder, V. N. Novikov, and S. C. Glotzer, Phys. Rev. E 66, 030101 (2002). 